\title{
Novel DOX-MTX Nanoparticles Improve Oral SCC Clinical Outcome by Down Regulation of Lymph Dissemination Factor VEGF-C Expression in vivo: Oral and IV Modalities
}

\author{
Mehran Mesgari Abbasi ${ }^{1,2}$, Amir Monfaredan ${ }^{3}$, Hamed Hamishehkar ${ }^{1}$, Khaled \\ Seidi $^{4}$, Rana Jahanban- Esfahlan ${ }^{2,4 *}$
}

\begin{abstract}
Background: Oral squamous cell carcinoma (OSCC) remains as one of the most difficult malignancies to control because of its high propensity for local invasion and cervical lymph node dissemination. The aim of present study was to evaluate the efficacy of novel $\mathrm{pH}$ and temperature sensitive doxorubicin-methotrexateloaded nanoparticles (DOX-MTX NP) in terms of their potential to change the VEGF-C expression profile in a rat OSCC model. Materials and Methods: 120 male rats were divided into 8 groups of 15 animals administrated with 4-nitroquinoline-1-oxide to induce OSCCs. Newly formulated doxorubicin-methotrexate-loaded nanoparticles (DOX-MTX NP) and free doxorubicin were IV and orally administered. Results: Results indicated that both oral and IV forms of DOX-MTX- nanoparticle complexes caused significant decrease in the mRNA level of VEGF-C compared to untreated cancerous rats $(p<0.05)$. Surprisingly, the VEGF-C mRNA was not affected by free DOX in both IV and oral modalities ( $p>0.05$ ). Furthermore, in DOX-MTX NP treated group, less tumors characterized with advanced stage and VEGF-C mRNA level paralleled with improved clinical outcome $(\mathbf{p}<0.05)$. In addition, compared to untreated healthy rats, the VEGF-C expression was not affected in healthy groups that were treated with IV and oral dosages of nanodrug $(p>0.05)$. Conclusions: VEGF-C is one of the main prognosticators for lymph node metastasis in OSCC. Down-regulation of this lymph-angiogenesis promoting factor is a new feature acquired in group treated with dual action DOX-MTX-NPs. Beside the synergic apoptotic properties of concomitant use of DOX and MTX on OSCC, DOX-MTX NPs possessed anti-angiogenesis properties which was related to the improved clinical outcome in treated rats. Taking together, we conclude that our multifunctional doxorubicin-methotrexate complex exerts specific potent apoptotic and anti-angiogenesis properties that could ameliorate the clinical outcome presumably via down-regulating dissemination factor-VEGF-C expression in a rat OSCC model.
\end{abstract}

Keywords: VEGF-C - DOX-MTX-NPs - oral squamous cell carcinoma (OSCC) - oral and IV modalities

Asian Pac J Cancer Prev, 15 (15), 6227-6232

\section{Introduction}

Oral squamous cell carcinoma (OSCC) ranks the sixth most common cancer worldwide as it accounts near to $95 \%$ of all oral neaoplasms and $38 \%$ of all head and neck cancers in particular tongue and lip (Kademani et al., 2005; Massano et al., 2006; Bell et al., 2007). In contrast to the good prognosis of lip cancers, tongue carcinomas generally exhibit a much more aggressive biological with an unfavorable prognosis and high metastatic potential (Jones et al., 1992; Kademani et al., 2005; Montoro et al., 2008). Unfortunately, the increase in incidence has not been paralleled by the development of new therapeutic agents while the survival rate has only improved slightly, with the 5-year survival rate stays $50 \%$ over the past 30 years. Patients with premalignant lesions and early stage cancers have a high rate of survival, but the vast majority of Stages III and IV cases are fatal (Zwetyenga et al., 2003; Bell et al., 2007; Rusthoven et al., 2010; Albano et al., 2013). The prognosis of SCC depends on a series of factors such as the proliferative activity of the tumor, degree of differentiation, and invasion and metastatic potential (Han et al., 2008).

Angiogenesis, the formation of new blood vessels from existing vasculature, is an important process in many malignancies including oral cancer (Zhao et al., 2013). It is the result of an intricate balance between pro-angiogenic and anti-angiogenic factors (Srivastava et al., 2014). The VEGF family is composed of several subtypes, including VEGF-A, VEGF-B, VEGF-C, and

${ }^{1}$ Drug Applied Research Center, ${ }^{2}$ Student Research Committee, ${ }^{4}$ Department of Medical Biotechnologies, Faculty of Advanced Medical Sciences, Tabriz University of Medical Sciences, ${ }^{3}$ Department of Hematology, Faculty of Medicine, Islamic Azad University, Tabriz, Iran*For correspondence: jahanbanr@tbzmed.ac.ir 
VEGF-D which exist as numerous splice variant isoforms (Sugiura et al., 2009). Shintani et a. ( 2004) described VEGF expression in OSCC, correlating subtypes A and $B$ with tumor angiogenesis and subtypes $C$ and $D$ with the risk of nodal metastases. Uehara et al. (2004) found a significant correlation between the high expression of VEGF in OSCC and worse prognosis. In view of this, the evaluation of VEGF-C and -D expression in oral SCCs could be a valuable tool for predicting their prognosis and designing agents with potential to inhibit their activity (Mohamed et al., 2004).

Combination chemotherapy and nanoparticle drug delivery are two fields that have shown substantial promise in cancer therapy. Administration of two or more drugs results in synergism effects between different drugs and can combat drug resistance through different mechanisms of action (Kalaria et al., 2009; Hu et al., 2010; Nasiri et al., 2013). Furthermore, nanoparticle drug delivery enhances therapeutic effectiveness whilst reduces side effects of the drug payloads by ameliorating their pharmacokinetics (Wang et al., 2010; Tacar et al., 2013; Salehi et al., 2014). Coupling these two active areas resulted in current measurable advances in improving the efficacy of cancer therapeutics (Mollazade et al., 2013). Although there are also some challenges and design specifications that need to be addressed in optimizing nanoparticle-based combination chemotherapy (Colleoni et al., 2002; Chen et al., 2011; Benival and Devarajan, 2012; Baykara et al., 2013; Lasrado et al., 2014).

Doxorubicin, as one of the most potent of the FDA approved chemotherapeutic Drugs has shown substantial anti-cancer potential, limited only by its cardiotoxicity (Deng and Zhang, 2013, Duong and Yung, 2013). However, combined to nanodelivery systems, DOXnanoparticles not only increase intracellular uptake of DOX, at the same time reduce its side effects significantly compared with conventional DOX formulations (Hu et al., 2010, Liboiron and Mayer, 2014) Methotrexate (MTX) is another central chemotherapeutic drug that is widely used either in monotherapy or in combination with other biologic and synthetic disease modifying anti cancer drugs (Rossi et al., 2010; Cipriani et al., 2014).

DOX-MTX NP is a new combination chemotherapy and nanoparticle drug delivery system that showed initial promising results in affecting the OSCC in rat model. However more studies require evaluating its efficacy, safety and also the mechanism of action.

In this respect, this study conducted to evaluate the efficacy of IV and oral modalities of DOX-MTX-loaded nanoparticles in term of their potential in affecting the expression level of VEGF-C since over-expression of this lymph-angiogenesis gene strongly associate with metastasis and poor prognosis. Targeting angiogenic pathway by combination chemotherapy and nanoparticle drug delivery may provide a promising approach for treatment of aggressive tumors including oral cancer.

\section{Materials and Methods}

Dual anticancer drug loaded nanoparticles

The synthesis procedure of nanoparticles was fully explained by Salehi et al(Salehi et al., 2014). Briefly, appropriate amount of novel synthesized nanoparticles were ultrasonically dispersed in the MTX solution for 5 min. After stirring for $24 \mathrm{~h}$ under dark conditions DOX$\mathrm{HCl}$ was added to MTX-loaded nanoparticles mixture and dispersed with the aid of ultrasonication (Sonics Vibra cell, Model: VCX 130 PB, Newton, CT) for $3 \mathrm{~min}$. The final carrier/drug ratio was 5 to 1 for both of drugs. The mixture was kept under magnetic stirring at room temperature for another $24 \mathrm{~h}$ under dark conditions. Then MTX-DOXloaded nanoparticles dispersion was left for $2 \mathrm{~h}$ to allow the sedimentation of the fine precipitates. DOX-MTXloaded nanocomposites were collected by centrifugation at $14000 \mathrm{rpm}$ for $15 \mathrm{~min}$ and vacuum dried for $24 \mathrm{~h}$ at room temperature and stored in a desiccators until used. The dual anticancer drug loaded nanoparticles were diluted with physiologic saline solution in appropriate concentration before administration to rats.

\section{Animals}

120 male Sprague-Dawley rats weighing $180 \pm 20$ grams were randomly divided into 8 groups. The animals were housed in the polycarbonate standard cages in a temperature-controlled animal room $\left(22 \pm 2^{\circ} \mathrm{C}\right)$ with a $12 / 12$ hours light/dark cycle during the experiments. The animals were provided by a standard rat pellet diet ad libitum. Drinking water containing 4-NQO was prepared three times a week by dissolving the carcinogen in distilled water and was given in light-opaque bottles.

\section{Experimental design}

120 rats randomly were divided into 8 groups of 15 animals each, as following: $i$ ) served as a carcinoma control and received 4-NQO(Sigma) at the concentration of 30ppm in their drinking water for 14 weeks without any treatment; $i$ i) - iii) served as the treatment groups and received 4-NQO at the concentration of 30ppm in their drinking water for 14 weeks and oral doses (Gavage) of Doxorubicin and the DOX-MTX-loaded nanoparticles respectively at the dose $5 \mathrm{mg} / \mathrm{kg}$ of body weight once a day on the days of 2,5 and 8 of the study; $i v$ ) $-v$ ) served as the treatment groups and received 4-NQO at the concentration of $30 \mathrm{ppm}$ in their drinking water for 14 weeks and intravascular (IV) dosages of doxorubicin and the DOX-MTX-loaded nanoparticles at the dose $1.5 \mathrm{mg} /$ $\mathrm{kg}$ of body weight once a day on the days of 2, 5 and 8 of the study; vi) and vii) served as the treated control group that received oral and IV the dose DOX-MTX-NPs $(5 \mathrm{mg})$ $\mathrm{kg}$ and $1.5 \mathrm{mg} / \mathrm{kg}$ of body weight once a day on the days of 2, 5 and 8 of the study, respectively); viii) served as normal control group and the rats of this group didn't get any carcinogen or treatment material; Death rate of the animals was also recorded during the study.

\section{Ethics}

All the ethical and the humanity considerations were performed according to the Helsinki humanity research declaration during the experiments and the euthanasia of the animals. All the animals' experiments were approved by the Ethics Committee of the Tabriz University of Medical Sciences. 


\section{Histological evaluation}

At the end of the interventional period, the animals were euthanized under anesthetic condition (Pentobarbital, $150 \mathrm{mg} / \mathrm{kg}$ IP). The tongue tissue samples were taken from each animal and were immediately fixed in $10 \%$ phosphate-buffered formalin. The $5 \mu \mathrm{m}$ thick microscopic sections were prepared after embedding of tissue samples in paraffin. Afterward, the sections were stained by Hematoxylin-Eosin staining method and histological evaluations were performed with light microscopy. Histopathological changes in tumors evaluated blindly by two pathologists.

Detection of VEGF-c mRNA expression by quantitative real time $P C R$

Briefly ,total RNA $(2 \mu \mathrm{g})$ extracted from homogenized fine powder of removed tongue tissues as described elsewhere (Jahanban Esfahlan et al., 2011). RNA were reverse transcribed to cDNA using Revert Aid first strand cDNA synthesis kit(fermentase). The resulting cDNA was diluted 1:30 fold and the PCR reaction was performed with $2 \mu 1 \mathrm{cDNA}, 10 \mathrm{pM}$ each forward and reverse primers, 12.5 $\mu 1$ SYBR Green PCR Master Mix (Fermentase) in a final volume of $25 \mu \mathrm{l}$. The thermal profile for the real-time Q-PCR was $95^{\circ} \mathrm{C}$ for $10 \mathrm{~min}$ and followed by 45 cycles of $95^{\circ} \mathrm{C}$ for 15 seconds and $60^{\circ} \mathrm{C}$ for $1 \mathrm{~min}$. The gene expression was expressed as fold change from the GAPDH level which is calculated as $2^{-\Delta \Delta C t}$. In addition, melting curve analysis was performed to assure the specificity of PCR product in this experiment. The following rat primers were used: VEGF-C (NM_053653.1): 5'- TTGTCTCTGGCGTGTTCCTTGC-3' (forward), 5' - A G G T C T T T G C C T T C G A A A C C C T T G - 3' (reverse). GAPDH (AF 106860): 5'-ATGACTCTA CCCACGGCAAG-3' (forward), 5'-CTGGAGATGGTGATGGGTT-3' (reverse).

\section{Data analysis}

The data were analyzed by SPSS 13 . One-Way Analysis Of Variance (ANOVA) was used to compare fold change differences of VEGF-c between and within studied groups followed by the multiple comparisons with the Tukey post-hoc test. Fischer's exact test used for analyzing pathological changes in groups. Chi square test used to verify the possible relation between expression of VEGF-c gene and pathological changes in tissue samples. A $p$ value $<0.05$ was considered significant.

\section{Results}

\section{Establishment of Oral Squamous Cell Carcinoma (OSCC)} model in rat

OSCC carcinogenesis usually develops through a multistep process that begins from hyperplasia and passes to mild, moderate and severe dysplasia before OSSC. 4-NQO induced OSCC have been used to study the various stages of oral carcinogenesis, because of its capability of inducing sequentially the phases of carcinogenesis (hyperplasia, mild dysplasia, moderate dysplasia, severe dysplasia, carcinoma in situ and OSSC). We have previously verified that 4-NQO successfully induces different stages of tongue carcinogenesis process in all cancer groups. High mortality rate, low weight gain, and frequency of OSCC and high proliferation severity of cancer control group compared to other groups demonstrate the efficacy of 4-NQO induced OSCC model (Mehdipour et al., 2013)

\section{Effect of DOX-MTX NP on mRNA expression of VEGF-C} in tongue tissues of OSCC rats models

Compared to all three healthy group, VEGF-C expressed approximately 2.98 folds more in cancerous group ( $\mathrm{p}=0.02)$ (Figure 1).

In groups that received oral doses $(5 \mathrm{mg} / \mathrm{kg}$ of body weight once a day on the days of 2,5 and 8 of the study), results showed that compared to untreated cancerous group, mRNA expression of VEGF-C decreased approximately 2.27 folds in DOX-MTX NPs treated groups $(p=0.046)$. At the other hand, no significant difference observed in VEGF-C expression between the

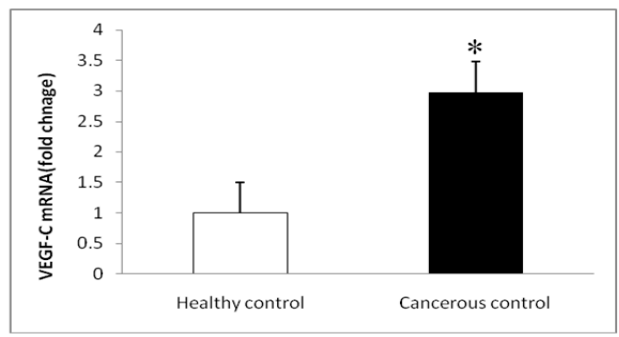

Figure 1. VEGF-C mRNA Expression in OSCC Cancerous Group and Healthy Control. Compared to healthy group, VEGF-c over-expressed $\sim 3$ fold in untreated cancerous group. ${ }^{*}$ Indicates to a significant $\mathrm{P}$ value $(\mathrm{p}<0.001)$

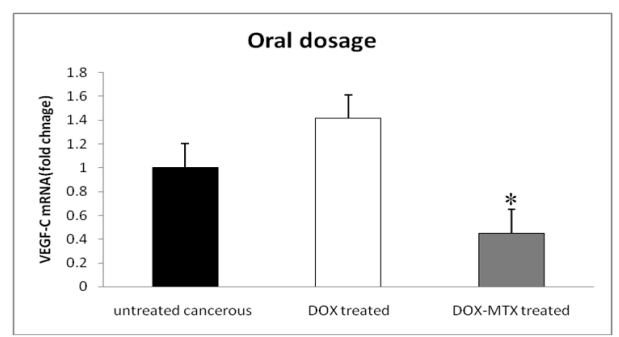

Figure 2. The Effect of Oral Dosage of DOX and DOXMTX NP on mRNA level of VEGF-C in OSCC Cancer Model in Rat. VEGF-C mRNA level decreased significantly in DOX-MTX NPs compared to untreated cancerous group whilst oral dosages of DOX could not alter amount of VEGF-c in treated group. *Indicate to a significant $\mathrm{P}$ value $(\mathrm{p}<0.05)$

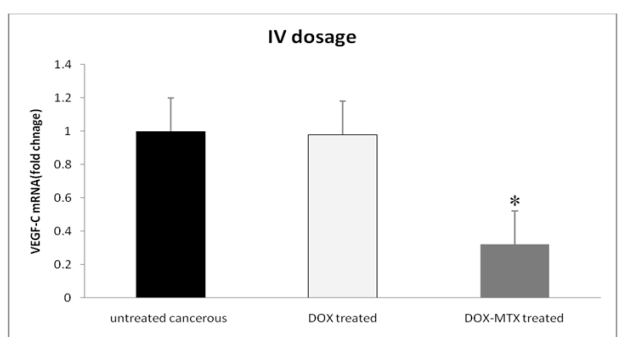

Figure 3. The Effect of IV Dosage of DOX and DOXMTX NP on mRNA level of VEGF-C in OSCC Cancer Model in Rat. VEGF-C mRNA level decreased significantly in DOX-MTX NPs compared to untreated cancerous group whilst IV dosages of DOX could not alter amount of VEGF-c in treated group. *Indicate to a significant $\mathrm{P}$ value $(\mathrm{p}<0.05)$ 
group that were received oral dosages of free DOX and untreated cancerous group $(\mathrm{p}=0.107)$ (Figure 2) (Fold changes represented as mean $\pm \mathrm{SE}$ ).

After IV treatment with DOX and DOX-MTX NP (1.5 $\mathrm{mg} / \mathrm{kg}$ of body weight once a day on the days of 2, 5 and 8 of the study), in DOX-MTX NP treated group, mRNA expression of VEGF-C decreased $\sim 3.33$ folds compared to untreated cancerous that was statistically significant $(\mathrm{p}=0.048)$, in contrast in DOX treated group, there was no significant decrease in VEGF-C mRNA level $(\mathrm{p}=0.943)$ (Figure 3)

Furthermore, we find no significant difference between IV and oral routes of DOX-MTX nanodrug $(\mathrm{p}=0.67)$ and neither DOX $(\mathrm{p}=0.173)$ (Figure 4).

All three healthy controls showed significant difference in VEGF-c MRNA expression compared to

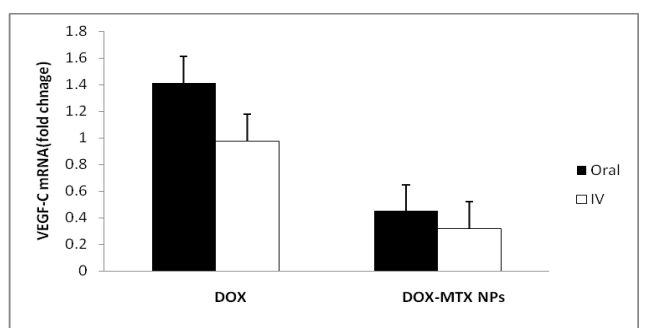

Figure 4. Comparison between the Efficacy of IV and Oral Administration of DOX-MTX NPin Affecting the VEGF-C mRNA Expression in OSCC Model in Rat. There was no difference between the oral and IV modalities in term of affecting the VEGF-C mRNA level in groups treated with DOX and DOX-MTX NPs. *Indicates to a significant $P$ value $(\mathrm{p}<0.05)$

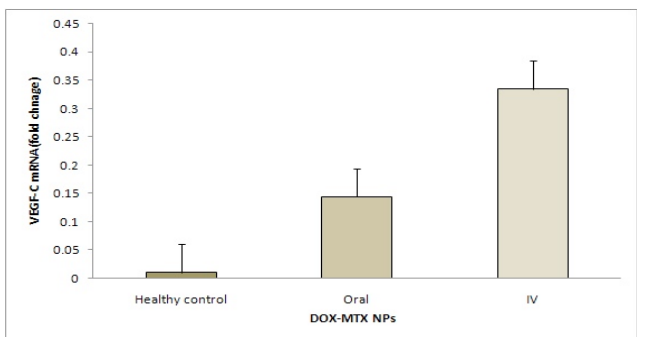

Figure 5. The Effect of Oral and IV Dosage of DOXMTX NP on mRNA Level of VEGF-C in Healthy Rats. There was no significant difference in VEGF-c amount of treated healthy in both modalities of nanodrug with that of untreated healthy control. *Indicates to a significant $\mathrm{P}$ value $(\mathrm{p}<0.05)$

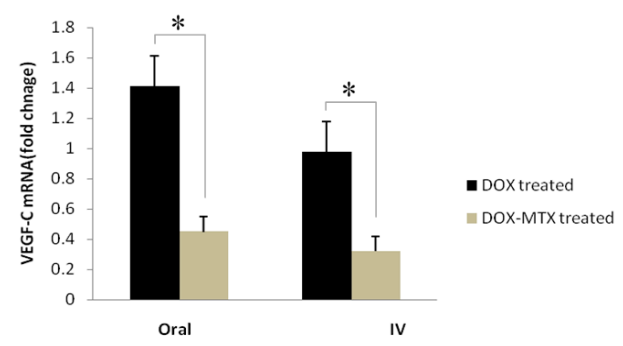

Figure 6. Comparison between the Efficacy of Oral and IV Dosage of DOX and DOX-MTX NP in Affecting the VEGF-c mRNA Expression in OSCC Model in Rat. Both Oral and IV route of DOX-MTX NP showed superior performance over DOX in affecting VEGF-C expression in OSCC model in rat. *Indicate to a significant $\mathrm{P}$ value $(\mathrm{p}<0.05)$
Table 1. High Stages of Tongue Cancer tend to Exhibit High Expression of VEGF-c While Low Level of VEGF-c in Samples Indicated for a Good Prognosis with Improved Clinical Outcome $(p=0.011$

\begin{tabular}{lrrrr}
\hline \multicolumn{4}{c}{ VEGF-C mRNA } \\
DOX-MTX NP & low & high & Total & P-value \\
\hline pathologic changes & & & & $\mathrm{p}=0.011$ \\
$\quad$ Low stage & $(85.7 \%) 12$ & $(0 \%) 0$ & $(85.7 \%) 12$ & \\
$\begin{array}{l}\text { High stage } \\
\text { Total }\end{array}$ & $(0 \%) 0$ & $(14.3 \%) 2$ & $(14.3 \%) 2$ & \\
\hline
\end{tabular}

untreated cancerous group $(\mathrm{P}>0.01)$. In case of evaluation of the safety of our nanodrug on normal cells and also its specificity to target tumoral cells, we included three healthy controls. Our results indicated that both healthy groups that were treated with oral and IV doses of DOXMTX NPs showed no significant difference in mRNA level of VEGF-C compared to untreated healthy group $(\mathrm{p}=0.285$ and $\mathrm{p}=0.634$, respectively) (Figure 5).

Our results indicate that both oral and IV modalities of MTX-DOX NPs exhibit superior performance over free DOX, in term of decreasing the VEGF-C mRNA level in OSCC model in rat ( $\mathrm{p}=0.047, \mathrm{p}=0.006$, respectively) (Figure 6).

Relation between VEGF-C mRNA level and histopatological changes

AS IV mode of nanodrug showed superior performance over oral form, hence this group subjected for evaluation of histopathological changes.

Our results showed that in DOX treated group $6 \backslash 13$ of lesion showed a low stage (No/Mild/moderate dysplasia) while $7 \backslash 13$ were advanced (Severe dysplasia, Carcinoma in situ and OSCC). At the other hand, we observed markedly increase in frequency of low stage tumors (12 14 vs 2\14) in group treated with IV doses of nonodrug. Pathologicl changes significantly were different between the two groups $(\mathrm{p}<0.05)$. Furthermore, no pathological changes detected in either of healthy controls, whilst all rats of cancerous group developed aggressive lesions.

Subsequently, we tested the relation between VEGF-C mRNA profile and the tumor stage in DOX and DOXMTX NP treated group. In this respect according to the observed mRNA fold changes, samples categorized in two main groups: group with high mRNA and group with low moderate mRNA level. As shown in Table 1, high stages of tongue cancer tend to exhibit high expression of VEGF-c while low level of VEGF-c in samples indicated for a good prognosis with improved clinical outcome $(\mathrm{p}=0.011)$

\section{Discussion}

Squamous cell carcinoma (SCC) is the most common head and neck cancer with poor clinical outcome (Montoro et al., 2008). One of the factors governing the poor prognosis of oral SCC is frequent metastasis of cancer cells to local lymph nodes or distant organs (Rusthoven et al., 2010). Combinatorial chemotherapy coupled with nanomedicine have opened appealing window to the current therapeutic approaches that always failed due to 
tumor cell resistance and unwanted side effects of drug on normal cells but by advent of nano-based drug delivery systems and combination chemotherapeutic agents this problem resolved drastically (Liboiron BD and Mayer LD, 2014)

Malignancies have the ability to induce growth of new blood vessels, which is important for tumor progression, aggressiveness, and ability to metastasize. It is a highly regulated to keep the intricate balance of pro and anti angiogeneic factors(Schliephake, 2003). Marked angiogenesis correlated well with the risk of nodal metastases and should probably imply a more aggressive postoperative adjuvant therapy (Shintani et al., 2004). Among VEGF families, it is known that VEGF-C and VEGF-D express on lymph nodes and promote nodal metastasis in OSCC (Shintani et al., 2004; Uehara et al., 2004). Moreover, OSCC angiogenesis correlates with $\mathrm{T}$ and $\mathrm{N}$ parameters and is an independent predictor of tumor recurrence and a reliable prognosticator (Zhao et al., 2013). In this respect, drugs that could inhibit activity of lymph node metastasis dissemination factors may provide promising approach for treatment of aggressive tumors including OSCC.

Cytotoxic effect of Doxurobicin most often attributed to its potential in induction of apoptosis by recruiting divergent targets (Gibson et al., 2005; Huang et al., 2011). However to our knowledge there is no record that indicate to the anti-angiogenesis potential of Doxorubicin in vitro or in vivo, nonetheless in our study, not oral and neither IV forms of DOX affect VEGF-C mRNA level in treated groups. Surprisingly in DOX treated groups even a subtle up- regulation of VEGF-C observed, although the difference was not statistically significant. Interestingly, both oral and IV forms of the new formulated DOX (DOX-MTX NP) showed high performance in affecting VEGF-c mRNA level and in particular this decrease in amount of VEGF-C paralleled with less aggressive tumors in this group.

In a single study related to anti-angiogentic properties of DOX, Fengjun Liu et al indicated to the role of DOX in inhibiting the VEGF expression in hepatocellular Carcinoma." Gene transfer of antisense HIF- $1 \alpha$ downregulated the expression of both HIF-1a and VEGF, whereas doxorubicin only downregulated VEGF expression. Both antisense HIF-1 $\alpha$ and doxorubicin inhibited expression of proliferating cell nuclear antigen, and combined to exert even stronger inhibition of proliferating cell nuclear antigen expression." (Liu et al., 2007; 2008). In this paper, Fengjun Liu et al clearly indicated to the feasible anti-angiogentic properties of DOX via modulating the expression of PCNA.

At the other hand, there are several studies that clearly indicate to the anti-angiogenesis properties of MTX. Methotrexate is a well-known effective therapeutic modality in the treatment of psoriasis. Apart from reducing the expression of CD31 (a known endothelial cell marker) in psoriatic lesions ,study by Olfat G. Shaker et al in 2013 shows that the percentage of clinical improvement in the examined psoriatic plaque was significantly positively correlated with the percentage of reduction in the amount of VEGF mRNA ( $r=0.850, p 0.001)$ and the percentage of reduction in the capillary perfusion (Shaker et al., 2013). Moreover Methotrexate inhibits in vitro corneal vascular endothelial cell proliferation and blocks VEGF- and bFGF-induced corneal neovascularization in vivo(Hirata et al., 1989). Others also demonstrated significant decrease in serum levels of VEGF after 2 and 6 months of treatment with low dose methotrexate and cyclophosphamide in patients with metastatic breast cancer (Colleoni et al., 2002) .In addition, neoadjuvant multidrug chemotherapy including high-dose methotrexate modifies VEGF expression in osteosarcoma (Rossi et al., 2010; Park et al., 2011).

We have previously shown that beside apoptotic effects, our nanodrug exerts potent anti proliferative and anti metastatic potentials, as well (Mesgari Abbasi et al., 2014, Mesgari Abbasi et al., 2014,Mesgari Abbasi et al., 2014.The anti-VEGF-C potential of DOX-MTX NP is an extra merit beside its enhanced apoptotic effects in its new format. These features makes DOX-MTX NP a powerful multifunctional weapon designed to combat resistant/ aggressive forms of a wide range of tumors whilst have least the cytotoxic effects on normal surrounding cells.

In conclusion, According to the obtained results, DOXMTX NPs is safe drugs that have the potential to inhibit activity of VEGF-C and improve the clinical outcome in invasive stages of OSCC.

\section{Acknowledgements}

This is a self financed study and we have received no financial support from elsewhere. However we'd like to acknowledge Drug Applied Research Center of Tabriz and also The Research Center of Tabriz International Hospital for their valuable technical support with Real Time PCR.

\section{References}

Albano PM, Lumang-Salvador C, Orosa J 3rd, et al (2013). Overall survival of Filipino patients with squamous cell carcinoma of the head and neck: a single-institution experience. Asian Pac J Cancer Prev, 14, 4769-74.

Baykara M, Buyukberber S, Ozturk B, et al (2013). Efficacy and safety of concomitant chemoradiotherapy with cisplatin and docetaxel in patients with locally advanced squamous cell head and neck cancers. Asian Pac J Cancer Prev, 14, 2557-61.

Bell RB, Kademani D, Homer L, Dierks EJ, Potter BE (2007). Tongue cancer: Is there a difference in survival compared with other subsites in the oral cavity? J Oral Maxillofac Surg, 65, 229-236.

Bell RB, Kademani D, Homer L, Dierks EJ , Potter BE (2007). Lipomer of doxorubicin hydrochloride for enhanced oral bioavailability. Int J Pharm, 423, 554-561.

Chen Y, Wan Y, Wang Y, Zhang H , Jiao Z (2011). Anticancer efficacy enhancement and attenuation of side effects of doxorubicin with titanium dioxide nanoparticles. Int $J$ Nanomedicine, 6, 2321-26.

Cipriani P, Ruscitti P, Carubbi F, Liakouli V, Giacomelli R (2014). Methotrexate in rheumatoid arthritis: optimizing therapy among different formulations. current and emerging paradigms. Clin Ther, 36, 427-35.

Colleoni M, Rocca A, Sandri MT, et al (2002). Low-dose oral methotrexate and cyclophosphamide in metastatic breast 
cancer: antitumor activity and correlation with vascular endothelial growth factor levels. Ann Oncol, 13, 73-80.

Deng Y, Zhang H (2013). The synergistic effect and mechanism of doxorubicin- $\mathrm{ZnO}$ nanocomplexes as a multimodal agent integrating diverse anticancer therapeutics. Int $J$ Nanomedicine, 8, 1835-41.

Duong HH, Yung LY (2013). Synergistic co-delivery of doxorubicin and paclitaxel using multi-functional micelles for cancer treatment. Int J Pharm, 454, 489-95

Gibson RJ, Bowen JM, Cummins AG , Keefe DM (2005). Relationship between dose of methotrexate, apoptosis, p53/ p21 expression and intestinal crypt proliferation in the rat. Clin Exp Med, 4, 188-95.

Han W, Yang X, Huang X, Hu Q, Wang Z (2008). Metastases to lingual lymph nodes from squamous cell carcinoma of the tongue. Br J Oral Maxillofac Surg, 46, 376-78.

Hirata S, Matsubara T, Saura R, Tateishi H, Hirohata K (1989). Inhibition of in vitro vascular endothelial cell proliferation and in vivo neovascularization by low-dose methotrexate. Arthritis Rheum, 32, 1065-73.

$\mathrm{Hu}$ CM, Aryal S, Zhang L (2010). Nanoparticle-assisted combination therapies for effective cancer treatment. Ther Deliv, 1, 323-34.

Huang WY, Yang P M, Chang YF, Marquez VE, Chen CC (2011). Methotrexate induces apoptosis through p53/p21dependent pathway and increases E-cadherin expression through downregulation of HDAC/EZH2. Biochem Pharmacol, 81, 510-17.

Jahanban Esfahlan R, Zarghami N, Jahanban Esfahlan A, et al (2011). The possible impact of obesity on androgen, progesterone and estrogen receptors (ER $\alpha$ and $E R \beta)$ Gene expression in breast cancer patients. Breast Cancer, 227-37.

Jahanban Esfahlan R, Zarghami N, Rahmati-Yamchi M, et al (2011). Quantification of steroid receptors gene expression in breast cancer patients: possible correlation with serum level of adipocytokines. J Cancer Therapy, 2, 659-65.

Jahanban Esfahlan R, Zarghami N, Valiyari S, et al (2012). Adiponectin can affect ER signaling in obese breast cancer patients. J Cancer Therapy, 3, 115-21

Jones KR, Lodge-Rigal RD, Reddick RL, Tudor GE , Shockley WW (1992). Prognostic factors in the recurrence of stage I and II squamous cell cancer of the oral cavity. Arch Otolaryngol Head Neck Surg, 118, 483-5.

Kademani D, Bell RB, Bagheri S, et al (2005). Prognostic factors in intraoral squamous cell carcinoma: the influence of histologic grade. J Oral Maxillofac Surg, 63, 1599-605.

Kalaria DR, Sharma G, Beniwal V, Ravi Kumar MN (2009). Design of biodegradable nanoparticles for oral delivery of doxorubicin: in vivo pharmacokinetics and toxicity studies in rats. Pharm.Res., 26, 492-501.

Lasrado S, Moras K, Pinto GJ ,et al. (2014). Role of concomitant chemoradiation in locally advanced head and neck cancers. Asian Pac J Cancer Prev, 15, 4147-52.

Liboiron BD, Mayer LD (2014). Nanoscale particulate systems for multidrug delivery: towards improved combination chemotherapy. Ther Deliv, 5, 149-71.

Liu F, Tan G, Li J, et al (2007). Gene transfer of endostatin enhances the efficacy of doxorubicin to suppress human hepatocellular carcinomas in mice. Cancer Sci, 98, 1381-7.

Liu F, Wang P, Jiang X, et al (2008). Antisense hypoxia-inducible factor 1alpha gene therapy enhances the therapeutic efficacy of doxorubicin to combat hepatocellular carcinoma. Cancer Sci, 99, 2055-61.

Massano J, Regateiro FS, Januario G , Ferreira A (2006). Oral squamous cell carcinoma: review of prognostic and predictive factors. Oral Surg Oral Med Oral Pathol Oral Radiol Endod, 102, 67-76.
Mehdipour M, Taghavi ZA, Mesgari AM, et al (2013). Evaluation of the effect of two systemic doses of HESA-A on prevention of induced tongue neoplasm in rats. J Dent Res Dent Clin Dent Prospects, 7, 218-24.

Mohamed KM, Le A, Duong H, et al (2004). Correlation between VEGF and HIF-1alpha expression in human oral squamous cell carcinoma. Exp Mol Pathol, 76, 143-52.

Mollazade M, Nejati-Koshki K, Akbarzadeh A, et al (2013). PAMAM dendrimers augment inhibitory effects of curcumin on cancer cell proliferation: possible inhibition of telomerase. Asian Pac J Cancer Prev, 14, 6925-28.

Montoro JR, Ricz HA, Souza L, et al (2008). Prognostic factors in squamous cell carcinoma of the oral cavity. Braz $J$ Otorhinolaryngol, 74, 861-6.

Nasiri M, Nejati NZ, Koshki K, et al (2013). Curcumin and silibinin inhibit telomerase expression in T47D human breast cancer cells. Asian Pacific J Cancer Prev, 14, 3449-3453.

Park SK, Kim HI, Yang YI, Hur DY (2011). Effects of methotrexate on vascular endothelial growth factor, angiopoietin 1, and angiopoietin 2 in nasal polyps. Am J Rhinol Allergy, 25, e129-e32.

Rossi B, Schinzari G, Maccauro G, et al (2010). Neoadjuvant multidrug chemotherapy including high-dose methotrexate modifies VEGF expression in osteosarcoma: an immunohistochemical analysis. BMC Musculoskelet Disord, 11, 34 .

Rusthoven KE, Raben D, Song JI, et al (2010). Survival and patterns of relapse in patients with oral tongue cancer. J.Oral Maxillofac.Surg., 68, 584-589.

Salehi R, Hamishehkar H, Eskandani M, Mahkam M, Davaran S (2014). Development of dual responsive nanocomposite for simultaneous delivery of anticancer drugs. J Drug Target, 22, 327-42.

Schliephake H (2003). Prognostic relevance of molecular markers of oral cancer--a review. Int J Oral Maxillofac Surg, 32, 233-45.

Shaker OG, Khairallah M, Rasheed HM, et al (2013). Antiangiogenic effect of methotrexate and PUVA on psoriasis. Cell Biochem.Biophys, 67, 735-42.

Shintani S, Li C, Ishikawa T, et al (2004). Expression of vascular endothelial growth factor A, B, C, and D in oral squamous cell carcinoma. Oral Oncol, 40, 13-20.

Srivastava VK, Gara Rk Fau, Rastogi N, et al (2014). Serum vascular endothelial growth factor-A (VEGF-A) as a biomarker in squamous cell carcinoma of head and neck patients undergoing chemoradiotherapy. Asian Pac J Cancer Prev, 15, 3261-65.

Sugiura T, Inoue Y, Matsuki R, et al (2009). VEGF-C and VEGF-D expression is correlated with lymphatic vessel density and lymph node metastasis in oral squamous cell carcinoma: Implications for use as a prognostic marker. Int J Oncol, 34, 673-80.

Tacar O, Sriamornsak P, Dass CR (2013). Doxorubicin: an update on anticancer molecular action, toxicity and novel drug delivery systems. J Pharm Pharmacol, 65, 157-70.

Uehara M, Sano K, Ikeda H, et al (2004). Expression of vascular endothelial growth factor and prognosis of oral squamous cell carcinoma. Oral Oncol, 40, 321-32.

Wang Y, Wei X, Zhang C, Zhang F, Liang W (2010). Nanoparticle delivery strategies to target doxorubicin to tumor cells and reduce side effects. Ther Deliv, 1, 273-87.

Zhao SF, Yang XD, Lu MX, et al (2013). Prognostic significance of VEGF immunohistochemical expression in oral cancer: a meta-analysis of the literature. Tumour Biol, 34, 3165-71.

Zwetyenga N, Majoufre-Lefebvre C, Siberchicot F, Demeaux H, Insolle J (2003). Squamous-cell carcinoma of the tongue: treatment results and prognosis. Rev Stomatol Chir Maxillofac, 104, 10-17. 